\title{
Caracterización de la influencia del factor visual en los pacientes con alteraciones del equilibrio
}

\author{
J. Rama-López, N. Pérez-Fernández
}

\author{
CHARACTERISATION OF THE INFLUENCE EXERTED \\ BY THE VISUAL FACTOR IN PATIENTS WITH BALANCE DISORDERS
}

\begin{abstract}
Summary. Introduction. Visual information provides data about the position and movement of the head with respect to objects and serves as a source of reference for the proper perception of verticality. It also plays a specific role in postural control by modifying the efficiency of the vestibular and proprioceptive responses. Aims. Our aim was to determine how visual information interacts with the symptoms of patients with vertigo, dizziness or instability and to find out the degree of disability it produces. Patients and methods. A study was conducted in 200 patients who had visited because of dizziness, vertigo, instability or loss of balance. We analysed the aspects related to the clinical symptoms of the patients. First, we examined the influence visual stimuli have on the symptoms of vestibular disease itself and their repercussions on the situation between crises. Second, the degree of disability was evaluated on the specific DHI (Dizziness Handicap Inventory) questionnaire. Results. As regards the influence exerted by visual stimulation on the main symptoms, $77.5 \%$ reported that it had no effect and $21 \%$ said that it made them worse. With regard to the influence of visual stimulation during the intercritical period, it was of no relevance in $53.5 \%$ of patients, $26.5 \%$ were unaffected, it had an exacerbating effect in $4 \%$ and in $7.5 \%$ it induced it. Different disorders were observed depending on the type of disease suffered by the patient, and disability was greater according to the influence exerted by the visual factor. Conclusions. Postural adjustments induced by visual stimuli are capable of triggering instability with serious consequences. [REV NEUROL 2004; 39: 513-6]
\end{abstract}

Key words. Balance. DHI. Dizziness. Instability. Vertigo. Visual factor.

\section{INTRODUCCIÓN}

Para el mantenimiento del equilibrio, la información visual aporta datos acerca de la posición y el movimiento de la cabeza con respecto a los objetos del entorno y es una fuente de referencia para la adecuada percepción de la verticalidad [1]. Se necesitan tanto la visión foveal como la periférica, aunque para el control postural esta última parece la más importante. En la interacción con otros sistemas, la información visual no se necesita absolutamente, como es fácil de considerar si se tiene en cuenta la capacidad para mantener el equilibrio con los ojos cerrados.

Además de la capacidad para reducir el balanceo, la visión desempeña un papel específico en el control postural modificando la eficiencia de las respuestas vestibulares y propioceptivas [2]. Un sujeto de pie en un entorno visual que se mueve hacia la derecha se desplaza o balancea en dicha dirección, ya que el movimiento del escenario se interpreta como un desplazamiento propio hacia la izquierda que exige un movimiento de corrección postural en dirección contraria. Por tanto, si la información visual y la vestibular-propioceptiva entran en conflicto, domina el estímulo visual. Ahora bien, la información visual puede malinterpretarse, ya que aisladamente es insuficiente para interpretar y diferenciar entre el movimiento de los objetos (exocéntrico) y el propio (egocéntrico), como ocurre al sentarse dentro de un coche a la par de otro que se empieza a mover. Sorprendentemente, estos fenómenos no son similares en todos los sujetos.

Recibido: 17.12.03. Recibido en versión revisada: 23.06.04. Aceptado: 05.07.04.

Departamento de Otorrinolaringología. Clínica Universitaria. Facultad de Medicina. Universidad de Navarra. Pamplona, Navarra, España.

Correspondencia: Dr. Nicolás Pérez. Dpto. ORL. Clínica Universitaria de Navarra. Avda. Pío XII, 36. E-31008 Pamplona (Navarra). E-mail: nperezfer @unav.es

(C) 2004, REVISTA DE NEUROLOGÍA
Uno de los trabajos más antiguos sobre la idiosincrasia en la percepción de la información visual relevante para el control postural es el de Asch y Witkin [3,4]. Estos autores encontraron que los sujetos normales se podían dividir en 'dependientes de campo' y en 'independientes de campo', de acuerdo a la capacidad para reorientarse a la verticalidad tomando en consideración el grado de inclinación del entorno visual, los primeros, y del propio grado de inclinación, los segundos. A los primeros también se les denominó 'dependientes visuales'. Este hecho es especialmente relevante en los pacientes con una afectación vestibular bilateral que en fase aguda poseen una especial sensibilidad a la estimulación visual, pero que, a medida que progresa la compensación vestibular, se apoyan más en la información propioceptiva y aprenden a ignorar las informaciones visuales erróneas. En los sujetos dependientes de campo, o dependientes visuales, esta transición puede no ocurrir y puede aparecer una muy baja tolerancia a las situaciones de conflicto visual, lo cual forma parte del síndrome de vértigo visual [5].

Este fenómeno de intolerancia al conflicto visual es común a varios síndromes, que además tienen un carácter 'seudoagorafóbico', como son la 'neurosis de la calle', el 'síndrome del supermercado', el 'síndrome de desorientación vestibular del motorista' [6], la 'fobia espacial' y el 'vértigo visual' [7]. Podemos decir que hablamos de un subgrupo de pacientes con una alteración vestibular parcialmente compensada o crónica, en los que la sintomatología vestibular es específica del contexto en el que se desenvuelve el sujeto [8]. Dicha especificidad situacional se relaciona con la estrategia de integración sensorial, en la que la información visual y somatosensorial completan la información vestibular deficitaria. Son pacientes especialmente vulnerables a la información visual inadecuada o deficiente y a la irregularidad de la superficie de soporte.

El objetivo de este estudio es determinar en pacientes con vértigo, mareo o inestabilidad cómo interactúa la información 
visual sobre su sintomatología y conocer el grado de discapacidad que ello produce.

\section{PACIENTES Y MÉTODOS}

Este estudio se realizó sobre una muestra de 200 pacientes vistos consecu tivamente por presentar cualquier tipo de enfermedad vestibular manifestada por mareo, vértigo, inestabilidad o desequilibrio. La edad media fue de $48,5 \pm 15,3$ años; el $41,5 \%$ correspondía a varones $(n=83)$ y el $58,5 \%$ a mujeres $(n=117)$. En el 78,5\% de los pacientes se pudo determinar el lado de la lesión (27\% en el oído derecho, $44 \%$ en el izquierdo y 7,5\% en ambos), mientras que en el $21,5 \%$ no era relevante la lateralidad del problema.

En el momento de la consulta se preguntó a los pacientes acerca del síntoma principal y la situación clínica en que se encontraban es ese momento. Junto a esto, se les pasó un cuestionario específico acerca de la influencia que tenía la información visual sobre su sintomatología, tanto en situación basal (intercrisis) como durante las crisis; las respuestas posibles fueron: no afecta, agrava, induce o no se aplica. Para valorar el grado de discapacidad, se les pidió que contestaran el cuestionario DHI (del inglés, dizziness handicap inventory) en su versión adaptada al español [9]. Además, se les realizó una exploración clínica otoneurológica y pruebas vestibulares complementarias consistentes en prueba calórica, pruebas rotatorias, posturografía dinámica y posturografía dinámica computarizada.

En el presente estudio únicamente pasamos a analizar los aspectos relacionados con la clínica de los pacientes. Por una parte, la influencia que el estímulo visual tiene, por un lado, en los síntomas propios de la enfermedad vestibular y, por otra, su repercusión en la situación entre crisis, si es que dicha enfermedad así evolucionase. En segundo lugar, el grado de discapacidad generado, atendiendo a una selección de cuatro ítems del DHI seleccionados, por cuestionar cada uno de ellos aspectos de interés a este estudio (Tabla I).

\section{Análisis estadístico}

Se empleó el programa estadístico SPSS, versión 10. Los pacientes se clasificaron de acuerdo al diagnóstico definitivo y por el efecto de la estimulación visual. Se realizó un estudio descriptivo de la muestra estudiada. Para analizar el efecto que la estimulación visual tenía en los diferentes pacientes incluidos en el estudio de acuerdo al diagnóstico definitivo se utilizó el test de Mann-Whitney, y para analizar las diferencias entre las puntuaciones del DHI de acuerdo al efecto que el estímulo visual tiene en fase intercrítica se utilizó el test no paramétrico de Kruskal-Wallis.

\section{RESULTADOS}

La distribución de los síntomas que eran motivo de la consulta fue la siguiente: vértigo en el 65\% $(n=130)$, inestabilidad en el 26,5\% $(n=53)$, mareo en el $6 \%(n=12)$ y desequilibrio en el $2 \%(n=4)$. Los diagnósticos obtenidos en los pacientes incluidos en el estudio se muestran en la tabla II.

La gravedad de los síntomas en el momento de la consulta fue descrito por los pacientes de la siguiente manera: 'afectado gravemente', el 6,5\% $(n=13)$; 'afectado moderadamente', el 63\% $(n=126)$, y 'bien' o asintomáticos en el momento de la consulta, el 30,5\% $(n=61)$.

Respecto a la influencia que la estimulación visual tenía sobre la sintomatología principal, el $77,5 \%$ refería que no le afectaba y el $21 \%$ que la agravaba. En cuanto a la influencia de la estimulación visual en la sintomatología durante el período intercrítico, en el 53,5\% de los pacientes no era relevante, por no existir en ese período de tiempo síntoma alguno, en el $26,5 \%$ no la afectaba, en el $4 \%$ la agravaba y en el $7,5 \%$ la inducía.

Se observó la existencia de una afectación diferente según el tipo de enfermedad que sufría el paciente. Realizando comparaciones múltiples dos a dos mediante el test de Mann-Whitney se obtuvieron diferencias estadísticamente significativas al analizar el grupo de pacientes con diagnóstico de enfermedad de Ménière o vértigo posicional frente al grupo de pacientes con diagnóstico de inestabilidad $(p<0,001)$. El número de pacientes que afirmaban que la estimulación visual tenía efecto en su clínica (respuestas: afecta, agrava o induce) era del $15 \%$ en los diagnosticados de enfermedad de Ménière, el 14\% de los pacientes con vértigo posicional y el 23\% de los pacientes con inestabilidad. No se obtuvieron diferencias significativas al comparar entre sí el resto de los grupos, cuyo tamaño de la muestra permitió realizar el test estadístico $(p>0,05)$.
Tabla I. Selección de preguntas del cuestionario DHI utilizada en este trabajo. A cada pregunta, el paciente responde 'sí', 'a veces', o 'no', y adjudica a cada una de estas respuestas 4, 2 y 0 puntos, respectivamente.

\begin{tabular}{ll}
\hline Item $^{\text {a }}$ & Pregunta \\
\hline P 4 & $\begin{array}{l}\text { Paseando por un pasillo o nave, } \\
\text { ise incrementa su mareo/vértigo? }\end{array}$ \\
\hline E 10 & $\begin{array}{l}\text { Por su mareo/vértigo, ¿le molesta } \\
\text { estar frente a otras personas? }\end{array}$ \\
\hline F 12 & Por su mareo/vértigo, ¿evita asomarse desde sitios altos? \\
\hline P 17 & Pasear por una acera, ¿incrementa su mareo/vértigo? \\
\hline
\end{tabular}

a En referencia a la escala completa original del DHI [9].

Tabla II. Distribución de diagnósticos

\begin{tabular}{ll}
\hline Enfermedad de Ménière & $n$ \\
\hline Vértigo posicional & 60 \\
\hline Inestabilidad & 45 \\
\hline Vértigo espontáneo recurrente & 36 \\
\hline Neurinoma del acústico & 29 \\
\hline Vértigo postraumático & 7 \\
\hline Otosclerosis & 6 \\
\hline Neuritis vestibular & 5 \\
\hline Fístula perilinfática & 5 \\
\hline Ototoxicidad & 4 \\
\hline Agorafobia & 2 \\
\hline
\end{tabular}

La puntuación media obtenida de las respuestas dadas a la versión reducida del DHI fue de $6,7 \pm 2,2$. Por grupos, de acuerdo a la influencia que la estimulación visual tenía durante la situación intercrítica, los resultados fueron los siguientes: en el que no se aplicaba ese efecto, $6,6 \pm 2,1$; en el que no afectaba, 6,3 $\pm 2,6$; en el que agravaba, $7,7 \pm 2,3$, y en el que inducía, $7,1 \pm 1,7$. Se obtuvieron diferencias estadísticamente significativas $(p=$ 0,042 ) en estos valores entre los grupos en los que la información visual no les afectaba frente a aquellos en los que agravaba o inducía su sintomatología (Figura). Las diferencias fueron especialmente significativas al analizar el grupo al que no afecta la información visual frente al grupo en el que la información visual desempeña algún papel sobre su sintomatología, obtenido al juntar los grupos en los que agrava o induce la sintomatología, en el que la puntuación media en el DHI es de 7,23 $\pm 1,76(p=0,02)$.

\section{DISCUSIÓN}

La existencia de síntomas vestibulares inducidos por la estimulación visual, más o menos compleja, es una realidad que se ha caracterizado recientemente. Se describe entre pacientes que han sufrido algún episodio de vértigo en el contexto de una enfermedad vestibular y que, de manera idiosincrásica, son dependientes visuales [10,11], y como estrategia postural adquirida después de la neurectomía vestibular en pacientes con enfermedad de Ménière [12].

En nuestro trabajo se realizó una anamnesis sistemática sobre este aspecto diferenciando la situación en la crisis de la exis- 


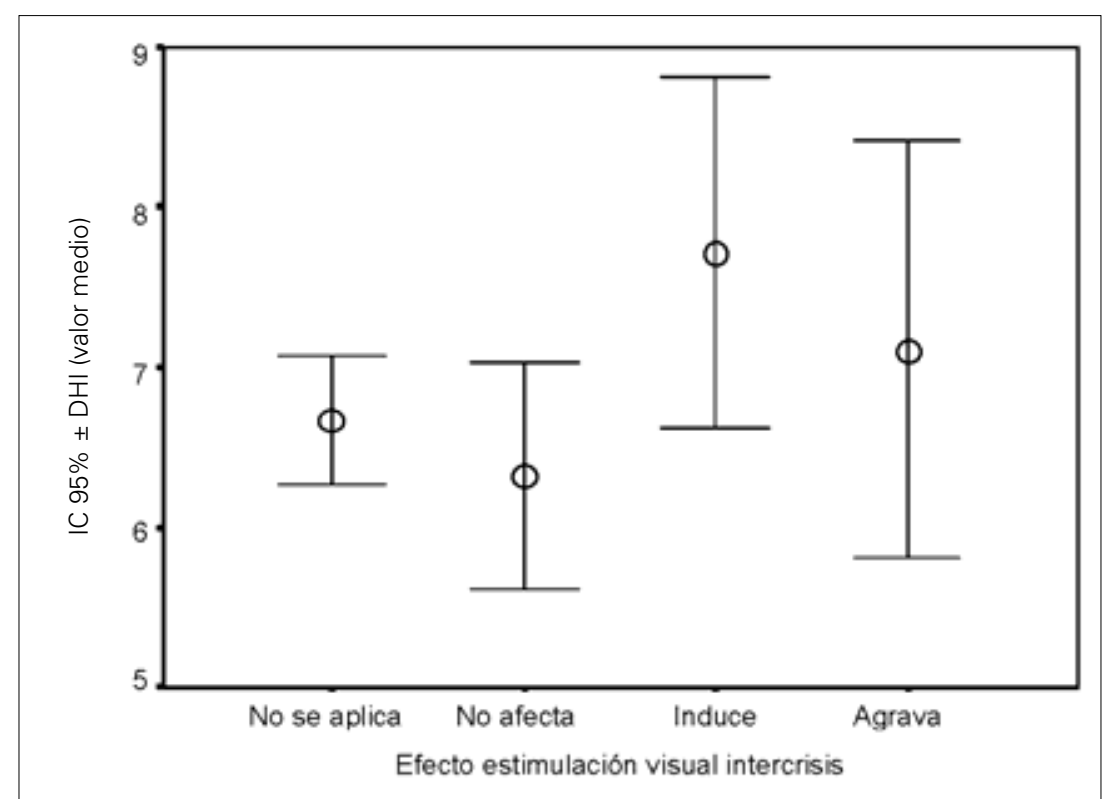

Figura. Valor medio en la versión reducida del DHI en los diferentes grupos de pacientes agrupados según la influencia del estímulo visual.

tente en el período intercrítico. Este último nos ha parecido de mayor interés y es el que pasamos a analizar.

La cifra de pacientes que refieren síntomas vestibulares inducidos o agravados por el estímulo visual es del 11,5\%. Los pacientes mencionaron como causante de este hecho una o más de las siguientes situaciones: la deambulación por sitios iluminados artificialmente en entornos visualmente muy estructurados (pasillos de supermercados, grandes centros comerciales), la observación de tráfico rodado desplazándose hacia o desde el paciente (durante la conducción) y desde el campo periférico (durante la espera para cruzar un paso de peatones o un transporte público), el desplazamiento sobre escaleras mecánicas y la apertura/cierre de puertas automáticas, entre otras. Nos parece oportuno explicar, en primer lugar, los fundamentos de este fenómeno y, en segundo, la relevancia del mismo.

La contribución de la visión en el mantenimiento de la postura depende de una serie de características como son la iluminación, la distancia entre el ojo y el objeto, el contraste, el tamaño y la frecuencia espectral del objeto [13]. En líneas generales, se puede considerar que la función estabilizadora de la información visual se basa en la capacidad que aporta para detectar el desplazamiento retiniano de las imágenes debido al movimiento involuntario de la cabeza con respecto al entorno visual.

Durante años, se ha considerado que la visión periférica o ambiental está particularmente mejor preparada para procesar correctamente la información visual útil en la orientación y equilibrio que la visión central [14-16]. Actualmente, parece más oportuno considerar que ambas son útiles, pero están segregadas en su función según la calidad o el tipo de información somatosensorial de la que el paciente dispone [17]. Así pues, cuando ésta es correcta y congruente, la información visual periférica y central son complementarias, mientras que cuando se modifica convenientemente la superficie de apoyo del paciente, con el objeto de alterar la información somatosensorial, la visión central es más eficaz en la regulación postural en el plano frontal, mientras que la periférica lo es en el anteroposterior. Ahora bien, no sólo es relevante para esta actividad estabilizadora la información visual aferente, sino también la oculomotora, lo cual explica el hecho observado de la mejoría en la estabilidad o reducción del balanceo corporal en pacientes con neuritis vestibular al reducir la intensidad o suprimir el nistagmo espontáneo [18]. En general, cuando un sujeto estable y quieto se expone a una estimulación que cubre todo el campo visual, se producen períodos alternativos de percepción de movimiento del sujeto (vección) y del objeto. La dirección de las correcciones posturales se alinea perfectamente con el plano de movimiento del estímulo y su magnitud es superior durante los períodos en los que domina la percepción de vección [19].

Podemos considerar que los ajustes posturales inducidos por el estímulo visual en condiciones más o menos desventajosas para el paciente son capaces de provocar una desestabilización con serias consecuencias. El número de pacientes en el que esto ocurre no es pequeño si tenemos en cuenta que hablamos de una población, como la aquí estudiada, con una gran variedad de enfermedades de tipo vestibular. Estos pacientes deben ser sujetos de un estudio más pormenorizado, para identificar los hechos que subyacen a esta particular sensibilidad. No sólo la funcionalidad del receptor periférico es relevante, sino también el grado de compensación vestibular logrado y el tipo de interacción visuovestibular existente, en todos los ámbitos posibles [11].

Por otra parte, este hecho debe reconocerse e identificarse en la anamnesis. Creemos que es preciso preguntar específicamente a los pacientes con mareo, vértigo o inestabilidad acerca de la influencia de la información visual en su estabilidad o incluso sobre los efectos que puede tener como desencadenante de un agravamiento de sus síntomas; este aspecto debe incluirse, como apuntan otros autores, en la creación de bases de datos de pacientes con trastornos del equilibrio [20]; ello, no sólo explica una porción importante de las alteraciones del paciente, sino que también guía una parte nada desdeñable del tratamiento cuando se indica seguir un protocolo de rehabilitación vestibular.

\section{BIBLIOGRAFÍA}

1. Shumway-Cook A, Woollacott M. Motor control. Theory and practical applications. 2 ed. Baltimore: Lippincott Williams and Wilkins; 2001.

2. Lee DN, Lishman JR. Visual propioceptive control of stance. Journal of Human Movement Studies 1995; 1: 87-95.

3. Asch SE, Witkin HA. Studies in space orientation. II. Perception of the upright with displaced visual fields and with the body tilted. J Exp Psychol 1948; 38: 455-77.
4. Witkin HA. The perception of the upright. Sci Am 1959; 200: 51-6.

5. Bronstein AM. Visual vertigo syndrome: clinical and posturography findings. J Neurol Neurosurg Psychiatry 1995; 59: 472-6.

6. Page NGR, Gresty MA. Motorist's vestibular disorientation syndrome. J Neurol Neurosurg Psychiatry 1985; 48: 729-35.

7. Furman JM, Jacob RG, Redfern MS. Clinical evidence that the vestibular system participates in autonomic control. J Vestib Res 1998; 8: 27-34. 
8. Jacob RG, Woody SR, Duncan DB. Disconfort with space and motion: a possible marker of vestibular dysfunction assessed by the situational characteristics questionnaire. J Psychopath Behav Assess 1994; 15: 299-324.

9. Pérez N, Garmendia I, Martín E, García-Tapia R. Adaptación cultural de dos cuestionarios de medida de la salud en pacientes con vértigo. Acta Otorrinolaringol Esp 2000; 51: 572-80.

10. Guerraz M, Yardley L, Bertholon P. Visual vertigo: symptom assessment, spatial orientation and postural control. Brain 2001; 24: 1646-56.

11. Bronstein AM. Visual and psychological aspects of vestibular disease. Curr Opin Neurol 2002; 15: 1-3.

12. Lacour M, Bathelemy J, Borel L. Sensory strategies in human postural control before and after unilateral vestibular neurotomy. Exp Brain Res 1997; 115: 300-10.

13. Bronstein AM, Guerraz M. Visual-vestibular control of posture and gait: physiological mechanisms and disorders. Curr Opin Neurol 1999; 12: 5-11.

\section{CARACTERIZACIÓN DE LA INFLUENCIA \\ DEL FACTOR VISUAL EN LOS PACIENTES \\ CON ALTERACIONES DEL EQUILIBRIO}

Resumen. Introducción. La información visual aporta datos acerca de la posición y el movimiento de la cabeza con respecto a los objetos y es fuente de referencia para la adecuada percepción de la verticalidad. Además, desempeña un papel específico en el control postural modificando la eficiencia de las respuestas vestibulares y propioceptivas. Objetivo. Determinar en pacientes con vértigo, mareo o inestabilidad cómo interactúa la información visual sobre su sintomatología y conocer el grado de discapacidad que produce. Pacientes y métodos. Se realizó el estudio de 200 pacientes vistos por mareo, vértigo, inestabilidad o desequilibrio. Analizamos los aspectos relacionados con la clínica de los pacientes. En primer lugar, la influencia que el estímulo visual tiene en los síntomas propios de la enfermedad vestibular y su repercusión en la situación entre crisis. En segundo lugar, el grado de discapacidad valorado por el cuestionario específico DHI (del inglés dizziness handicap inventory). Resultados. Respecto a la influencia que la estimulación visual tiene sobre la sintomatología principal, el 77,5\% refería que no le afectaba, y el 21\%, que le agravaba; en cuanto a la influencia de la estimulación visual durante el período intercrítico, en el $53,5 \%$ de los pacientes no era relevante, en el $26,5 \%$ no la afectaba, en el $4 \%$ la agravaba y en el 7,5\% la inducía. Se observó la existencia de una afectación diferente según el tipo de enfermedad que sufría el paciente, y una mayor discapacidad según la influencia del factor visual. Conclusiones. Los ajustes posturales inducidos por el estímulo visual son capaces de provocar una desestabilización con serias consecuencias. [REV NEUROL 2004; 39: 513-6]

Palabras clave. DHI. Equilibrio. Factor visual. Inestabilidad. Mareo. Vértigo.
14. Schneider GE. Two visual systems. Science 1969; 163: 895-902.

15. Brandt T, Dichgans J, Koenig E. Differential effects of central versus peripheral vision on egocentric and exocentric motion perception. Exp Brain Res 1973; 16: 476-91.

16. Paulus WM, Starube A, Brandt T. Visual stabilization of posture. Physiological stimulus characteristics and clinical aspects. Brain 1984; 107: 1143-63.

17. Nougier V, Bard CH, Fleury M, Teasdale N. Contribution of central and peripheral vision to regulation of stance. Gait Posture 1997; 5: 34-41.

18. Jahn K, Strupp M, Krafczyk S. Suppression of eye movements improves balance. Brain 2002; 125: 2005-11.

19. Thurrell AEI, Bronstein AM. Vection increases the magnitude and accuracy of visually evoked postural responses. Exp Brain Res 2002; 147: 558-60

20. López-Gentili LI, Kremenchutzky M, Salgado P. Base de datos para pacientes con trastornos del equilibrio. Rev Neurol 2003; 36: 960-5.

\section{CARACTERIZAÇÃO DA INFLUÊNCIA \\ DO FACTOR VISUAL EM DOENTES \\ COM ALTERAÇÕES DO EQUILÍBRIO}

Resumo. Introdução. A informação visual faculta dados acerca da posição e movimento da cabeça relativamente aos objectos é éfonte de referência para a adequada percepção da verticalidade. Adicionalmente, desempenha um papel específico no controlo postural modificando a eficiência das respostas vestibulares e proprioceptivas. Objectivo. Determinar em doentes com vertigens, náusea ou instabilidade, de que modo interage a informação sobre a sua sintomatologia e conhecer o grau de incapacidade que produz. Doentes e métodos. Realizou-se com o estudo de 200 doentes observados por enjoo, vertigens, instabilidade ou desequilíbrio. Analisámos os aspectos relacionados com a sintomatologia dos doentes. Em primeiro lugar, a influência que o estímulo visual possui sobre os sintomas próprios das doença vestibular e sua repercussão na situação entre as crises. Em segundo lugar, o grau de incapacidade avaliado pelo questionário específico DHI (do inglês dizziness handicap inventory). Resultados. Relativamente à influência que o estímulo visual possui sobre a sintomatologia principal, 77,5\% referia que não era afectada e $21 \%$ que a agravava. Quanto à influência da estimulação visual durante o período intercrítico, em $53,5 \%$ dos doentes não era relevante, em $26,5 \%$ não a afectava, em $4 \%$ a agravava e em $7,5 \%$ a induzia. Observou-se a existência de um envolvimento diferente segundo o tipo de doença que o doente apresentava e uma maior incapacidade segundo a influência do factor visual. Conclusões. Os ajustes posturais induzidos pelo estímulo visual são capazes de provocar uma desestabilização com consequências graves. [REV NEUROL 2004; 39: 513-6]

Palavras chave. DHI. Enjoo. Equilíbrio. Factor visual. Instabilidade. Vertigens. 